\title{
Lectura grupal e infografías en la enseñanza y el aprendizaje de contenidos de divulgación científica en el contexto universitario
}

\author{
Diego F. Becerra-Rodríguez ${ }^{\star *}$, Carlos H. Barreto-Tovar ${ }^{2}$, Cesar A. Bernal-Torres ${ }^{3}$ y Andrés F. Ordoñez \\ (1) Centro de Tecnologías Para La Academia - CTA, Universidad de La Sabana, Chía, Cundinamarca, Colombia. \\ (correo-e: diego.becerra2@unisabana.edu.co) \\ (2). Facultad de Educación, Universidad de La Sabana, Chía, Cundinamarca, Colombia. \\ (correo-e: carlos.barreto2@unisabana.edu.co) \\ (3) Escuela Internacional de Ciencias Económicas y Administrativas- Universidad de La Sabana, Chía, Cundinamarca, \\ Colombia. (correo-e: cesar.bernal@unisabana.edu.co) \\ (4) Facultad de Ciencias, Universidad Antonio Nariño, Bogotá D.C., Colombia. (correo-e: andres.ordonez@uan.edu.co) \\ * Autor a quien debe ser dirigida la correspondencia.
}

Recibido Jun. 24, 2020; Aceptado Ago. 24, 2020; Versión final Oct. 22, 2020, Publicado Abr. 2021

\begin{abstract}
Resumen
El objetivo de esta investigación es analizar la importancia de la lectura grupal y la construcción colaborativa de infografías en el proceso de enseñanza y aprendizaje de los contenidos de los libros de divulgación científica en estudiantes universitarios. La investigación es de tipo cualitativo y de orden descriptivointerpretativo. Se realizó un experimento con 10 estudiantes de tercer semestre de medicina que cursaron una asignatura de física. Los resultados indican que los estudiantes participantes del estudio mostraron una mejora significativa de su aprendizaje de temas científicos. Esto contrasta de forma positiva con los resultados de los procesos de enseñanza y aprendizaje de este tipo de contenidos que utilizan estrategias pedagógicas con énfasis en aprendizajes memorísticos y de resolución de ejercicios de forma individual. Se concluye que la lectura grupal y el uso de las infografías para el contenido de textos de ciencia y tecnología son una práctica que permite realizar ejercicios de indagación que favorecen el aprendizaje conceptual de los estudiantes.
\end{abstract}

\section{Group reading and infographics in teaching and learning scientific information in the university context}

\begin{abstract}
The objective of this research study is to assess the importance of group readings and collaborative infographic construction in the process of teaching and learning scientific book contents to university students. This research study is qualitative and of descriptive-interpretive nature. An experiment is performed with ten thirdsemester medical students taking a physics course. The results show significant improvement in learning scientific topics by using group readings and collaborative infographic construction when compared to learning by using pedagogical strategies that put emphasis on memory and practice exercises that are solved individually. It is concluded that group reading and infographic use for teaching science and technology are practices that allow implementing inquiring-type activities that favor conceptual learning in students.
\end{abstract}




\section{INTRODUCCIÓN}

La divulgación científica implica el desarrollo de actividades experimentales, académicas y literarias, cuyo objetivo principal es trasladar los conocimientos científicos del mundo académico a toda la sociedad en general, para lograr entender el impacto de la ciencia y la tecnología en la sociedad y, reconocer que la apropiación social de la ciencia favorece el desarrollo y creación de nuevas ramas de ésta (Souza, 2016). Dentro de las distintas formas de divulgación científica está la literatura académica (Gans et al, 2017) que, junto con las actividades experimentales recreativas, son relevantes para las ciencias naturales.

Por otro lado, la formación en ciencias debe buscar la construcción de un aprendizaje articulado y en constante evolución (Khishfe y Lederman, 2006). Es decir que, si uno de los objetivos es lograr que los estudiantes adquieran una comprensión amplia de los principales fenómenos del mundo natural, es pertinente que el docente promueva en ellos el uso de estrategias que le faciliten el desarrollo de un pensamiento analítico y crítico, como es el caso de la lectura grupal y la construcción colaborativa de infografías (Alrwele, 2017). Según Rivas y Telleira (2009) este objetivo se puede lograr mediante el uso de la lectura, ya que ésta permite realizar predicciones, verificar hipótesis, y reflexionar acerca los planteamientos y aplicaciones científicas. Asimismo, es posible determinar que, si bien los estudiantes universitarios no carecen de hábitos de lectura, no existe una relación clara entre dichas lecturas y los que estas pueden aportar en su futura vida profesional, lo cual limita el desarrollo de una formación integral (Beckert y Gurgel, 2005) además, según Knight y Thompson (2020) la inclusión de textos en contextos de enseñanza de las ciencias se posiciona como un campo interesante y a la vez poco investigado.

Debido a lo anterior, en esta investigación, se muestra una propuesta del uso de las infografías en el aula como una estrategia para la enseñanza- aprendizaje, y que para este caso, está centrada en distintos libros de una colección de literatura de divulgación científica de la editorial Fondo de Cultura Económica de México, el principal, "Electromagnetismo: De La Ciencia A La Tecnología" Braun (1992) acompañado de la lectura y aspectos relevantes de otros textos de la misma editorial como "Michael Faraday: Un Genio De La Física Experimental" de Carmona et al (1995). "La Física en la Medicina". De Piña, M. (1998) "El Desarrollo De La Tecnología. La aportación De la Física”. De Alba (1993) y "Cacería de Cargas". De Piña (1995).

El objetivo de tomar como recurso educativo principal de investigación en el aula el libro "Electromagnetismo: De La Ciencia A La Tecnología" se justifica en una articulación y transversalidad de los fundamento teóricos y las aplicaciones tecnológicas del Electromagnetismo, así mismo, este recurso permite hacer un trabajo coordinado tanto con los libros complementarios seleccionados de la serie leamos la ciencia para todos, con la definición de conceptos, relación de variables o el desarrollo de actividades experimentales en el aula, propias de un curso de electricidad y magnetismo. Una de las características que más sobresalen del libro es que no solo muestra los conceptos apropiadamente sino que aborda también la relación de variables de los fenómenos sin la necesidad de la realización de ejercicios netamente de física, los cuales, en ocasiones solo promueven el trabajo centrado en la memorización de fórmulas sin comprender sus aplicaciones, la visión que se tomó del libro Electromagnetismo: De La Ciencia A La Tecnología fue una visión en la que este fuera el recurso de aprendizaje principal de todo el curso.

\section{OTROS ANTECEDENTES}

Según Bradshaw y Porter (2017) una infografía es “...una forma única de presentar información sintetizada de forma visual. Las infografías efectivas son atractivas, crean interés y llevan a la audiencia más información..." (p. 2). de igual forma, Souza (2016) comenta que la infografía configura un nuevo idioma de significar la información, ya que mediante la presentación concisa y optimizada de un texto se permite simplificar la explicación de procesos complejos que pueden abordar hechos históricos y conceptos científicos que en ocasiones son difíciles de comprender por los estudiantes. De igual forma, según Baglama et al (2017) éstas son de mucha utilidad para presentar información que es complicada de entender por medio del texto y su impacto en la sociedad cobra cada vez más validez, ya que una buena parte de ésta lee menos cada día, pero si está al pendiente de la información y la representación de datos mediante gráficas. Sin embargo, se debe tener en cuenta que el construir infografías requiere considerar las necesidades del público, para así comunicar los conceptos de la mejor manera, atendiendo a las preguntas de qué, quién, cuándo, dónde y cómo, de igual forma se aconseja que la infografía sea sencilla, completa, ética, con buen diseño, redacción e información veraz (Fazdil, 2018).

En las diferentes investigaciones que vinculan el uso de la infografía en la enseñanza (Alrwele; 2017; Baglama et al, 2017; Fazdil, 2018) se comenta que dentro de las ventajas que ofrece el uso de ésta en la formación universitaria, está el favorecer la comprensión de conceptos que pueden ser complejos para los estudiantes ya que al incluir textos e imágenes y mostrar de manera rápida y entretenida las temáticas, se facilita la construcción del conocimiento, sin embargo, en la literatura científica no es posible encontrar investigaciones 
que relacionan el uso de ésta en la enseñanza de disciplinas como la física, lo cual refuerza los planteamientos de Polman y Gebre (2015) al considerar que aún es necesario realizar más investigaciones que revelen las implicaciones de éstas en el aprendizaje de los estudiantes.

Así mismo, en un estudio realizado por Minervini (2016) en el cual se utiliza la infografía como recurso didáctico en el aula para la enseñanza de la Biología en estudiantes de quinto año, se menciona que el uso de éstas permite una mejor comprensión de procesos como la clonación de animales, la fabricación de antibióticos o las consecuencias y las causas de las enfermedades. En la investigación se utilizó infografías ya realizadas para la explicación de "El Proyecto Genoma Humano" que es un tema contemplado en los planes de estudio para ese curso y al mismo tiempo es actual y complejo. El desarrollo de su estrategia se dio en tres pasos, el primero fue un acercamiento al tema con el apoyo de una infografía de la temática, un segundo paso, de profundización de la temática alrededor de la infografía y con retroalimentación continua de los docentes y finalmente un tercer paso que recopila los resultados mediante una evaluación. Dentro de las ventajas encontradas con el uso de las infografías en el aula está que los gráficos ayudan más a la comprensión de conceptos y el hecho de trabajar de una manera más centrada en los estudiantes y con la utilización de este tipo de recursos didácticos potencia el trabajo colaborativo en el aula.

Teniendo en cuenta que, el objetivo de la educación se ha transformado y no se centra en la memorización de información, sino en la comprensión del entorno y la relación adecuada con éste mediante la integración de conocimientos, (Valderrama y Valderrama, 2014; Lahnwong, 2019), para esta investigación se ha planteado el desarrollo de un curso de Biofísica que a diferencia de los tradicionales no se centra en la resolución de ejercicios y memorización de fórmulas, sino en actividades de lectura de textos de divulgación científica y el desarrollo de infografías como herramienta de consolidación conceptual, partiendo de la concepción que de los estudiantes que poseen una buena capacidad de lectura, se espera que maximicen sus habilidades críticas y de conocimiento (Nuryana et al, 2020) y que las situaciones de aprendizaje por medio de la lectura, suscitan que el acto de aprender tenga una relación íntima con las oportunidades de construir y articular un significado de aplicación profesional de los conceptos. Además de esto, con el desarrollo de esta investigación se propone una alternativa de enseñanza que contribuya a transformar el modelo de recepción-transmisión predominante en la enseñanza universitaria, cuyas clases magistrales son el método principal, propiciando en los estudiantes un aprendizaje pasivo y de baja motivación.

\section{METODOLOGÍA}

Esta es una investigación de tipo descriptivo-interpretativo, en la cual se plantea el uso de la lectura grupal y las infografías para el proceso de enseñanza aprendizaje durante el desarrollo de un curso de física a nivel universitario para estudiantes de medicina, partiendo del conocimiento que, en general, la bibliografía utilizada para el desarrollo de los cursos de biofísica se centra en la solución de problemas principalmente de Física (Muñoz, 2012).

\section{Población y materiales}

La información, del trabajo de campo analizada en esta investigación, se obtuvo de un grupo de 10 estudiantes de un curso de Biofisica aplicada a la Medicina de una universidad privada con sede en la ciudad de Bogotá D.C.- Colombia. Del grupo de estudiantes 6 fueron hombres y 4 mujeres, con edades entre 18 y los 20 años, de ellos, 7 estudiantes cursaban la asignatura por primera vez y 3 la repetían. La razón por la que se seleccionó este curso para el uso de la lectura grupal y el diseño de las infografías como estrategias de enseñanza aprendizaje, obedece a que, históricamente en ese curso se ha evidenciado bajo rendimiento del aprendizaje por parte de los estudiantes que cursan esa asignatura, debido en particular a que, los estudiantes manifiestan tener pocos hábitos de lectura y no encontrar una fuerte relación de sus lecturas académicas con su futuro profesional, a pesar de que, los contenidos del curso están diseñados para mostrar las aplicaciones del electromagnetismo en la medicina.

Para el ejercicio de la lectura y el diseño de las infografías se utilizó el libro "Electromagnetismo: De La Ciencia A La Tecnología" con el propósito de evaluar los resultados obtenidos en términos de la correcta comprensión conceptual por parte de los estudiantes en temáticas de Electricidad y Magnetismo. La inclusión del libro como recurso didáctico se escogió bajo la premisa que, para proponer tareas de lectura en el aula, el docente debe seleccionar previamente el texto que mejor se adapte a los propósitos de aprendizaje del curso. Resaltando que la propuesta pretendió ir más allá de hacer una lectura en clase como actividad pasiva de aprendizaje, o como actividad complementaria en la que los estudiantes solo leen el texto en sus hogares y posteriormente se les solicita un resumen o un ensayo de éste, atendiendo a la propuesta de Probosari et al (2019) al considerar que las actividades de aprendizaje asociadas a la lectura no se deben hacer de manera espontánea, sino que se deben planear de forma estratégica y gradual. 
Por otro lado, es de resaltar que los libros de divulgación escogidos, no se centran en la solución de ejercicios como se hace en múltiples textos de física universitaria, sino que además cuenta el contexto histórico y social en el que se dieron los avances del electromagnetismo, también muestra sus aplicaciones tanto en la medicina como en la sociedad, lo cual, según Reis et al (2001) podría beneficiar el desarrollo de habilidades de razonamiento, contribuyendo a una mejor comprensión de los conceptos científicos.

Asimismo, es importante el carácter que dan los autores en la aplicabilidad de los avances científicos como las antenas y las comunicaciones inalámbricas, en aras de dar continuidad a esa interesante propuesta y hacer una fusión de lo encontrado en "La Física en la Medicina" y "Electromagnetismo: De La Ciencia A La Tecnología", tratando de conservar el carácter de llevar la ciencia para todos se solicitó a los estudiantes realizar una actividad complementaria a las clases, en la que los estudiantes investigan el funcionamiento, desde el sentido físico, y determinan qué utilidad encontrarán en sus aplicaciones y labores en sus vidas cotidianas como futuros médicos del país, dichos artefactos los utilizarán en exámenes que realizarán y son el Ecógrafo, el Cardiógrafo, los Rayos X, El Examen de Efecto Doppler y Tomografías.

\section{Instrumento}

Con el propósito de evaluar el impacto de la lectura grupal y el uso de las infografías en el aprendizaje de los contenidos del curso por parte de los estudiantes, una vez desarrollado el primer tercio de esos contenidos, se aplicó una prueba (pretest) con ese propósito y que también, sería el referente comparativo con los resultados de una prueba final (postest) del aprendizaje de los demás contenidos desarrollados en ese curso. La prueba inicial durante el primer tercio del desarrollo de los contenidos del curso tuvo el objetivo de identificar el grado de aprendizaje por parte de los estudiantes, cuando se tiene poca familiaridad del uso de la lectura grupal y las infografías para su aprendizaje; mientras que, la prueba final se hizo para evaluar el aprendizaje una vez se había realizado amplio uso de estas estrategias para su aprendizaje.

El formato de evaluación inicial (pretest) y final (postest) fue de selección múltiple con única respuesta, en la cual se tomó en cuenta los conceptos abordados en el aula mediante el desarrollo de las infografías y estuvo compuesto por 17 preguntas que fueron tomadas de los conceptos, relaciones de las variables 0 afirmaciones tratadas en el texto de la Ciencia Para Todos. Estas preguntas fueron aportadas por profesores del departamento de Física de la universidad donde se realizó el estudio, y que pretenden hacer parte de una evaluación formativa en la enseñanza de la física en cuanto a la comprensión de conceptos, relación de variables de distintos fenómenos y el funcionamiento de diferentes artefactos de la vida cotidiana que para su funcionamiento requieren de avances científicos y tecnológicos, en este caso relacionados con la electricidad y el magnetismo. Las pruebas (prestes y postest) estuvieron enfocados a conceptos como la Fuerza Eléctrica, Campo Eléctrico, Potencial Eléctrico, Campo Magnético entre otros. Al test propuesto se le determinó una fiabilidad con el alfa de Cronbach de 0,612, lo cual representa una fiabilidad aceptable según (HernándezSampieri \& Mendoza, 2018).

\section{Método}

La investigación se desarrolló en 35 sesiones de dos horas cada una, en el curso de Biofísica II, programado durante el periodo intersemestral - diciembre de 2019 y enero de 2020. Sesiones que siempre tuvieron lugar en horas de la mañana y fueron orientadas por el mismo docente. En general, las actividades del curso se desarrollaron en las siguientes fases:

\section{Fase 1: Introducción al curso, primera sesión de clase}

Se contextualizó a los estudiantes acerca de los objetivos, los conceptos, las actividades propuestas para el desarrollo del curso y los criterios de evaluación (presentación del syllabus). También, se ambientó a los estudiantes sobre el uso de las infografías como estrategia de enseñanza y aprendizaje y para eso se asignó de forma aleatoria los capítulos del libro seleccionado para el curso (3 por cada estudiante).

\section{Fase 2: Lectura grupal, abordaje de conceptos y construcción de infografías}

Los 10 estudiantes trabajaron en la lectura de los capítulos asignados. Esta fase se desarrolló en 31 sesiones, de las cuales, en cada sesión se destinó 1 hora para la lectura grupal de cada capítulo y 1 hora para la construcción de la infografía correspondiente. Es importante aclarar que, para cada sesión, cada estudiante debía realizar de forma autónoma la lectura de cada capítulo de la clase y en cada sesión, el estudiante responsable del capítulo se encargó de liderar la lectura del mismo y la construcción de la infografía, resaltando que, durante la lectura grupal se identificaban conceptos estructurantes del curso y las aplicaciones de la física en la medicina, posteriormente, se presentaba la infografía al grupo consolidando la articulación de la misma con los conceptos abordados en la sesión. 
En esta fase, algunas temáticas del curso fueron abordadas con otros textos de divulgación científica afines con los contenidos del curso, además, como complemento a la lectura de algunos capítulos, se desarrollaron actividades experimentales con el fin de relacionar los aspectos teóricos y prácticos de la temática, una de ellas fue la realización de una actividad experimental en una sesión den 2 horas del curso, para la comprensión de La Ley de Inducción de Faraday.

El abordaje conceptual de las cargas eléctricas y la naturaleza de la materia se hizo utilizando el libro "Cacería de Cargas" Piña (1995) trabajando el capítulo I. ¿Qué son las Cargas? analizando la carga como una propiedad intrínseca de la materia, identificando cuál es su unidad de medida y resaltando las circunstancias bajo las cuales se produce la atracción y repulsión entre cargas eléctricas, posteriormente se hizo un trabajo de aula aprovechando el glosario que se encuentra en él texto, para abordar conceptos como la ionización, líneas de campo y bandas de conducción. Igualmente, la definición de conceptos como la Fuerza Eléctrica, Campo y Potencial Eléctrico se desarrolló mediante la lectura grupal del libro "La Física en la Medicina" Piña (1998) que en su capítulo III, Sistema Nervioso, no se limita a mostrar las relaciones fuerza-carga y fuerzadistancia en la Ley de Coulomb, sino que permite que los estudiantes se apropien de los conceptos nombrados propiciando que el curso tenga sus características plenas de BIO-Física, articulando la parte Eléctrica con el cuerpo humano y mencionando la aplicación de los fundamentos teóricos de este núcleo temático aplicados en el contexto profesional futuro de los estudiantes. El trabajo de lectura activa y participativa de este libro fue muy importante para el desarrollo de la propuesta, porque permitió saber rol de la física (Electricidad) en el funcionamiento del cuerpo humano, permitiendo abordar en el aula temáticas como la comunicación entre neuronas o la determinación del potencial de membranas, todas estas cuestiones encajaron perfectamente en la articulación del curso de Biofísica Il para estudiantes de Medicina con la serie de La Ciencia para todos, dándole así una aplicación de aprendizaje real en el aula del carácter para todos.

\section{Fase 3: Análisis y evaluación de la apropiación conceptual}

Se realizó la finalización de la propuesta, aplicando el postest en una sesión de 2 horas, con el fin de evaluar la apropiación conceptual de los estudiantes, la articulación entre los conceptos abordados en la investigación y la relación de estos con su desempeño profesional con elementos cotidianos de ciencia para todos.

\section{Desarrollo de la propuesta}

Luego de lograr que los estudiantes comprendieran qué es una infografía y sus características, se pasó a tener en cuenta las ventajas encontradas sobre el uso de éstas en la enseñanza y el aprendizaje de las ciencias (Biología) sin embargo, para este caso particular no se llevó al aula infografías ya realizadas por algún autor, en primera instancia porque no se encontró en la literatura científica un repositorio de infografías desarrolladas del libro y la física en general, y en segunda instancia, porque la intención era que los mismos estudiantes fueran trabajando de manera colaborativa en la construcción de una infografía por cada capítulo, pretendiendo dar así un carácter al trabajo de lectura grupal un poco más activo y no contemplar el libro como estrategia pasiva de lectura, que puede generar desmotivación en los estudiantes al abordar un libro solo por leerlo como actividad de clase y luego, realizar un resumen o un ensayo de éste.

Teniendo en cuenta que los conocimientos sobre las Ciencias Naturales se pueden configurar en herramientas que permiten comprender la naturaleza (Defago y Ithuralde, 2018), vale la pena resaltar que la intención de la propuesta no es evaluar si este tipo de actividades (resumen o ensayo) son benéficas o no en la enseñanza de la física, solo es resaltar que el trabajo con un libro en las clases de física se puede abordar de otras formas, y a partir de estas otras formas de trabajo, tratar de dar un carácter más didáctico a los libros y de paso permitir determinar en qué medida los gráficos informativos desarrollados por los mismo estudiantes, impactan en las formas de acceso, construcción y apropiación de su conocimiento, así mismo se trata de utilizar la cultura visual con la que convive la sociedad actual y para este caso en particular de los estudiantes de Medicina participantes del estudio. Se trató de que ellos mismos explicaran con sus palabras los acontecimientos, conceptos y aplicaciones descritas en el libro de la Ciencia para todos, así mismo se trató de que ellos situaran a los autores relevantes en los avances científicos y tecnológicos de la electricidad y el Magnetismo en un contexto determinado tanto histórico como académico.

Considerando que el aprendizaje de las ciencias se considera un proceso que permite la construcción de posturas críticas del entorno con base en la evidencia científica (Cuvi et al, 2013) tras la actividad planeada alrededor del libro "Electromagnetismo: De La Ciencia A La Tecnología" Braun (1992) se utilizó una afirmación encontrada en el capítulo, Faraday. La Inducción Electromagnética, cuando se menciona que "...Mientras mayor sea el cambio del flujo, mayor será el valor de la corriente eléctrica que se inducirá. De esta forma nos damos cuenta de que se pueden lograr valores muy altos de corriente eléctrica con sólo cambiar el flujo magnético..." y luego compararla con otra afirmación del Capítulo IV. La Ley De Inducción Y La Relatividad del libro "Michael Faraday: Un Genio De La Física Experimental" Carmona et al (1995) "...En particular, el 
efecto de inducción dependía únicamente del movimiento relativo entre el circuito donde aparecía la corriente eléctrica y el imán, fuente del campo magnético..."

Desde el punto de vista científico, las dos afirmaciones son ciertas y en pocas palabras se refieren a lo mismo, entonces, para abordar el aspecto conceptual y la relación de variables de la Ley de inducción de Faraday se propuso que los estudiantes no solo se quedaran con el desarrollo de la infografía del capítulo, sino que se elaboraron unas actividades experimentales alrededor de este tema y, se solicitó a los estudiantes que tomaran una bobina de 1000 vueltas y otra de 12000 vueltas conectadas a un galvanómetro y a una interfaz Casylab que les permite analizar la corriente inducida, luego se les pidió que: 1) Tomaran un imán que trasladaron continuamente alrededor (por fuera) de la bobina, tomaran apuntes de lo que notaban en las mediciones del galvanómetro y lo mostrado en la interfaz. 2) Trasladaran continuamente (de forma horizontal) el imán dentro de la bobina, que tomaran apuntes de lo que observaban y qué mencionaran las diferencias encontraban en las mediciones del galvanómetro y la interfaz en comparación de cuando se traslada el imán por fuera de la bobina. 3) Finalmente se hicieron los mismos pasos anteriores, pero con una bobina de 12000 vueltas y los estudiantes sacaron sus propias conclusiones acordes con la teoría del Electromagnetismo.

Durante el desarrollo de la actividad, fue llamativo ver a los estudiantes reconociendo qué es y cómo funciona un galvanómetro, ya que gracias a los elementos de laboratorio con los que contó la investigación fue posible, no solo realizar ejercicios de lectura que contextualizan ampliamente sobre el instrumento, sino también poder trabajar con ello, después de la actividad experimental. Al igual que, en la publicación de Collazos et al (2016), los estudiantes demostraron mejores argumentos para socializar y sustentar los resultados, en este caso, comprobaron la relación de variables que existen en torno a la Ley de Inducción de Faraday, con los datos encontrados analizaron qué sucede si la bobina tiene más o menos espiras, o qué sucede si no hay variación en el flujo magnético, siendo esto posible a partir de las afirmaciones del libro y seguir un camino experimental alrededor de éstas, que sirvió para relacionar la teoría con la práctica.

\section{RESULTADOS}

Los resultados de este estudio se presentan en dos componentes: ganancia de aprendizaje y factores que se pudieron observar en el aula.

\section{Ganancia de aprendizaje}

El primer componente abordado en el análisis de los resultados es la determinación de la ganancia de aprendizaje de los estudiantes, calculadas mediante el factor de Hake, esto, teniendo en cuenta los conocimientos previos de los estudiantes y los resultados al final del trabajo centrado en el libro en el que se basa la propuesta. Hake (1998) en su trabajo propone una expresión matemática para calcular la ganancia relativa del aprendizaje en estudiantes que presentan evaluaciones de tipo selección múltiple por medio factor $g$ de Hake, de acuerdo a los resultados obtenidos se podrá concluir si efectivamente la propuesta de trabajo centradas alrededor del libro y su metodología fueron efectivas, la expresión matemática para determinar $g$ es:

$$
g=\frac{(\% \text { Postest })-(\% \text { Pretest })}{100-(\% \text { Pretest })}
$$

De acuerdo a los resultados obtenidos se establece tres rangos de medida de la ganancia de aprendizaje: i) Zona de ganancia alta sí el factor de Hake cumple con $g \geq 0,7$; ii) Zona de ganancia media sí el factor de Hake cumple con $0,3 \leq g<0,7$; iii) Zona de ganancia baja sí el factor de Hake cumple con $g<0,3$. Para establecer el nivel de ganancia de aprendizaje y analizar si las actividades propuestas fueron efectivas en cuanto a un nivel de correcta apropiación conceptual por parte de los estudiantes, se utilizó el parámetro antes mencionado, analizando la cantidad de respuestas acertadas y erróneas de las pruebas (pretest y postest) en el grupo de estudiantes. Las pruebas fueron calificadas sobre un puntaje de 5,88 puntos por cada pregunta acertada, de tal manera que, si un estudiante respondió correctamente en el pretest y el postest, su resultado será de 100 puntos. La Tabla 1 muestra los resultados generales de cada uno de los estudiantes para la prueba inicial y final de los contenidos del curso y respectiva la ganancia del aprendizaje.

Tabla 1. De los resultados general y, por cada estudiante del curso en el pretest y postest.

\begin{tabular}{|l|c|c|c|c|}
\hline Estudiante & Pretest & Postest & Ganancia de aprendizaje & Nivel de ganancia de aprendizaje \\
\hline Estudiante 1 & 26 & 71 & 0,61 & Media \\
\hline Estudiante 2 & 30 & 94 & 0,91 & Alta \\
\hline Estudiante 3 & 34 & 77 & 0,65 & Media \\
\hline
\end{tabular}


Tabla 1: continuación

\begin{tabular}{|l|c|c|c|l|}
\hline Estudiante & Pretest & Postest & Ganancia de aprendizaje & Nivel de ganancia de aprendizaje \\
\hline Estudiante 4 & 40 & 82 & 0,62 & Media \\
\hline Estudiante 5 & 40 & 78 & 0,63 & Media \\
\hline Estudiante 6 & 60 & 88 & 0,70 & Alta \\
\hline Estudiante 7 & 40 & 94 & 0,90 & Alta \\
\hline Estudiante 8 & 26 & 88 & 0,83 & Alta \\
\hline Estudiante 9 & 30 & 71 & 0,59 & Media \\
\hline Estudiante 10 & 22 & 71 & 0,62 & Media \\
\hline Promedio del grupo & 34,8 & 81,4 & & \\
\hline
\end{tabular}

Se toman los promedios mostrados anteriormente para determinar la ganancia de Hake,

$$
g=\frac{(81,4)-(34,8)}{100-(34,8)}=\frac{46,6}{65,2}=0,71
$$

Del anterior resultado se puede observar que el valor de $g$ para el grupo de 10 estudiantes es de 0,71 , lo cual configura una ganancia de aprendizaje alta, de igual forma el $60 \%$ de los estudiantes obtienen ganancias de aprendizaje Media y el $40 \%$ restante ganancias de aprendizaje Altas, dando relevancia a la investigación realizada y demostrando su efectividad pedagógica en cuanto al manejo conceptual de los estudiantes.

\section{Factores observados en el aula}

Adicional, a los resultados del aprendizaje de los contenidos del curso, en el desarrollo de la investigación se pudo observar en los estudiantes cambios cualitativos relacionados con la motivación hacia el aprendizaje y las actividades propuestas para la clase. En la medida en que se avanzó en el desarrollo del curso fue cotidiano escuchar, de parte de los estudiantes, expresiones que denotaron la motivación en las actividades y el trabajo colaborativo en las sesiones de clase como "Me gusta aprender Física de una forma diferente y sin tanta fórmula" y "Es interesante saber que lo que aprendemos en aula tiene aplicaciones en contextos de nuestro futuro desempeño profesional". Esto permiten evidenciar en los estudiantes una actitud favorable para su aprendizaje y el aprecio por los contenidos relacionados con resultados de la investigación.

Por otro lado, luego de realizar un comparativo entre las infografías de los contenidos de los primeros y los últimos 7 capítulos del libro, se observó por parte del profesor y los propios estudiantes, una mejora significativa de las infografías de los últimos capítulos en relación con las de los primeros en aspectos de éstas, tales como: a) la calidad en el diseño, en particular en sus elementos gráficos, el adecuado uso de colores, y la presentación general de las infografías finales se caracterizan por tener un mejor aspecto, ser más completas y más ilustrativas a la vista, b) los desarrollos conceptuales, en este se aspecto se logró encontrar que mientras en las infografías iniciales se resaltaban los conceptos relevantes de los capítulos trabajados, a lo largo del curso y en las infografías finales, donde no solo se hacía esto mismo, se resaltan los conceptos relevantes y también se establecen interrelaciones entre los conceptos de distintos capítulos, resaltando incluso una mayor cantidad y calidad de los conceptos abordados, transformándolos así en conceptos estructurantes del curso que permiten identificar distintas aplicaciones de los contenidos del curso al contexto profesional del grupo, y c) las competencias comunicativas de los estudiantes, al evidenciarse un mejor uso del lenguaje verbal, la fluidez y la comprensión de la lectura del grupo en general, esto porque, mientras, al inicio de la propuesta era necesario leer un párrafo entre dos y tres veces para lograr la comprensión del texto, en las últimas sesiones del curso la lectura y la compresión fue más fluida y dinámica.

También, los resultados de este estudio permitieron identificar que, en comparación con grupos de estudiantes que cursaron previamente la asignatura mediante la metodología de enseñanza tradicional, el grupo que participó en esta investigación muestra mejores resultados en cuanto a la apropiación conceptual y diferencias significativas en la adecuada ejecución de procedimientos y en la recordación de fórmulas. Finalmente, en cuanto a la diferencia en los resultados del pre y postest de algunos estudiantes, se considera que esta puede ser debida a factores como el estilo de aprendizaje y los rasgos característicos y propios de cada estudiante, los cuales influyen en los procesos de aprendizaje, de igual forma, con la consolidación y calidad de las infografías. También, se observó que en algunos estudiantes la construcción de estas tuvo más dificultad, esto se pudo generar debido a las capacidades individuales relacionadas con el dibujo, la comprensión de lectura, la organización de la información y la creatividad. 


\section{DISCUSIÓN}

A partir de los resultados obtenidos se puede establecer que la propuesta de lectura grupal, complementada con la construcción colectiva de infografías, no solo es efectiva para aprender aspectos complejos de las ciencias y transformarlos para generar conocimiento en audiencias más amplias tal como comenta Souza (2016), sino que, también es efectiva en términos pedagógicos, ya que se logra evidenciar una estructura conceptual apropiada en los estudiantes, traducida en una ganancia grupal de aprendizaje alta, de igual forma, los resultados del estudio son coherentes con las posturas de Khoury et al (2019) al resaltar que los recursos gráficos, en este caso infográficos, tienen la oportunidad de aumentar el atractivo y la adecuada comprensión del conocimiento científico, permitiendo que el conocimiento de las ciencias naturales llegue a públicos a los que por sí solos no tendrían la posibilidad de tener un impacto positivo.

También, los hallazgos de la investigación coinciden con investigaciones que señalan que cuando los estudiantes construyen infografías con temas de su interés, es este caso la física en su proyección profesional, se permite obtener un mayor compromiso por parte de ellos en la construcción de su propio conocimiento (Polman y Gebre, 2015). De igual forma, se concuerda con que el uso de las infografías en la educación no solo configura nuevas estrategias didácticas de enseñanza, sino que beneficia el aprendizaje de los estudiantes incluso si tienen dificultades en la apropiación del conocimiento (Alrwele, 2017; Baglama et al, 2017; Fazdil, 2018; Ozdamli et al, 2016) y también permiten determinar mejoras en sus aprendizajes.

Con el desarrollo de la investigación es posible corroborar los resultados reportados por Beckert y Gurgel (2005) cuando comentan que la lectura grupal de textos de ciencia y tecnología es una práctica que permite realizar ejercicios de indagación que favorecen no solo el aprendizaje conceptual de los estudiantes, sino que también les permite establecer relaciones entre las concepciones previas sobre una temática y lo que pueden aprender con el texto. Además, se resalta que, con el desarrollo de la investigación es posible generar en los estudiantes y la sociedad hábitos de lectura con base en temas de ciencia, de igual forma, es posible potenciar la lectura en la sociedad, y no solo por parte de quien desarrolle el ejercicio (maestro) sino que a su vez, como en este caso, se replica la lectura de todos los libros en otros 10 estudiantes, multiplicando así su impacto. De igual forma, con la ejecución de la propuesta se demostró que en la enseñanza de la física es posible desarrollar actividades de aula diferentes a la exposición de conceptos, memorización de fórmulas y desarrollo repetitivo de ejercicios, así mismo se pudo notar que en el momento que los estudiantes realizan sus actividades de manera colaborativa trabajan con más motivación en las actividades propuestas para la clase.

Por otro lado, como prospectiva de la investigación, se considera interesante realizar un segundo ejercicio que tome dos grupos, un grupo experimental con el cual se desarrolle la misma metodología de actividades de lectura y construcción de infografías en pro de indagar no solo la apropiación conceptual sino como comenta (Alrwele, 2017) identificar también el desarrollo de habilidades de investigación, compromiso escolar, motivación y autoconfianza, y contrastar estos resultados con otro grupo de control en el cual se desarrollen actividades cotidianas de aula, para luego comparar los resultados de esos dos grupos analizando cuál obtiene mejores ganancias en los aprendizajes de conceptos de Electricidad y Magnetismo.

\section{CONCLUSIONES}

De acuerdo con el trabajo presentado y a los resultados obtenidos, se pueden plantear las siguientes conclusiones principales:

1.- La lectura grupal y el uso de las infografías para el contenido de textos de ciencia y tecnología son una práctica que permite realizar ejercicios de indagación que favorecen el aprendizaje conceptual de los estudiantes, y permite establecer relaciones entre las concepciones previas sobre una temática y lo que pueden aprender con la lectura del texto.

2.- En la enseñanza de contenidos científicos es posible desarrollar actividades de aula mediante el uso de infografías que fomentan la comprensión lectora de los estudiantes, aportan a sus competencias comunicativas y no están centradas en la exposición de conceptos, memorización de fórmulas y desarrollo repetitivo de ejercicios.

3.- La lectura grupal y construcción colaborativa de infografías, propicia que el grupo de estudiantes demuestre una ganancia de aprendizaje alta, la cual se refleja en la construcción de una estructura conceptual apropiada, relación de variables y la correcta solución de problemáticas en temas generales de los contenidos analizados en las clases.

\section{REFERENCIAS}

Alrwele, N., Effects of Infographics on Student Achievement and Students' Perceptions of the impacts of Infographics, doi: 10.15640/jehd.v6n3a12, Journal of Education and Human Development, Vol. 6, No. 3, pp. 104-117 (2017) 
Alba, F., El Desarrollo De La Tecnología. La aportación De la Física, 1ª edición, La ciencia Para Todos, Fondo de Cultura Económica, México D.F. (1993)

Beckert, E. y Gurgel, C., La lectura de un texto como estrategia de investigación del pensamiento CTS: Las visiones de los futuros profesores de Bilogía, Revista Eureka sobre Enseñanza y Divulgación de las Ciencias, 2 (2), 141-154 (2005)

Baglama, B. Yucesoy, Y. Uzunboylu, H. y Özcan, D., Can infographics facilitate the learning of individuals with mathematical learning difficulties?, doi: 0.5937/IJCRSEE1702119B, International Journal of Cognitive Research in Science, Engineering and Education, 5 (2), 119-128 (2017)

Braun, E., Electromagnetismo: De la ciencia a la Tecnología, 1aㅡ edición, La ciencia Para Todos, Fondo de Cultura Económica, México D.F. (1992)

Bradshaw, M. y Porter, S., Infographics: A New Tool for the Nursing Classroom, doi: 10.1097/NNE.0000000000000316, Nurse Education, 42 (2), 57-59 (2017)

Carmona, G., y otros 10 autores, Michael Faraday: Un Genio De La Física Experimental. 1ª edición, La ciencia Para Todos, Fondo de Cultura Económica. México D.F. (1995)

Collazos, C. Otero, H. Isaza, J. y Mora, C., Diseño y Construcción de una Máquina de Wimshurst para La Enseñanza de la Electrostática, doi: 10.4067/S0718-50062016000500011, Formación Universitaria, 9(5), 107-116 (2016)

Cuvi, N. Geirgii,C. Guarderas, P. y Arce, M., El camarote de Darwin: un Club de Lectura para aprender sobre la vida de Charles Darwin y su teoría de la evolución, Revista Eureka sobre Enseñanza y Divulgación de las Ciencias 10(2), 219$233(2013)$

Defago, A. y Ithuralde, R., El Diseño Curricular de Química del Ciclo Superior de la Educación Secundaria en la provincia de Buenos Aires, Argentina: una posible lectura para las aulas, Revista Eureka sobre Enseñanza y Divulgación de las Ciencias 15(1), 1203 (2018)

Fadzil, H., Designing infographics for the educational technology course: Perspectives of preservice science teachers, Journal of Baltic Science Education, 17 (1), 8-18 (2018)

Gans, J. Murray, F. y Stern, S., Contracting over the disclosure of scientific knowledge: Intellectual property and academic publication, doi: 10.1016/j.respol.2017.02.005, Research Policy, 46 (4) 820-835 (2017)

Hake, R., Interactive-engagement versus traditional methods: A sixthousand-student survey of mechanics test data for introductory physics courses, doi: 10.1119/1.18809, American Journal of Physics 66, 64-74 (1998)

Hernández-Sampieri, R., y Mendoza, C., Metodología de la Investigación, McGraw-Hill Interamericana, Ciudad de México (2018)

Khishfe, R. y Lederman, N., Teaching nature of science within a controversial topic: Integrated versus nonintegrated, doi: https://doi.org/10.1002/tea.20137, Journal of Research in Science Teaching, 43 (4), 395-418 (2006)

Khoury, C., Kisel, Y., Kantar, M., y otros 15 autores, Science-graphic art partnerships to increase research impact, doi: 10.1038/s42003-019-0516-1, Communications Biology 2, 295 (2019)

Knight, S., y Thompson, K., Developing a Text-Integration Task for Investigating and Teaching Interdisciplinarity in Science Teams, doi: 10.1007/s11165-020-09937-7, Research in Science Education, (2020)

Lahnwong, C., The Framework Development for Interpretation of Students Reading Literacy in Teaching and Learning Through Open Approach, doi: 10.1088/1742-6596/1340/1/012040, Journal of Physics: Conference Series, 1340 (1), 25, Article number 012040 (2019)

Minervini, M., La Infografía como recurso didáctico, Revista Latinoamericana de Comunicación Social, 27 (53), $103-127$ (2016)

Muñoz, J., Vales, M., y Cassiba, R., Porqué es necesaria una didáctica de la Biofísica. Anales de las Asociación Física Argentina, 23 (1), 20-3, Montevideo, (2012)

Nuryana, Z., Suroyo, A., y otros 3 autores, Literation movement for leading schools: Best practice and leadership power, doi: 10.11591/ijere.v9i1.20279, International Journal of Evaluation and Research in Education, 9 (1) 227 233 (2020)

Ozdamli, F., Kocakoyun, S., Sahin, T., y Akdag, S., Statistical reasoning of impact of infographics on education, doi: 10.1590/2176-457323502, Procedia Computer Science, 12th International Conference on Application of Fuzzy Systems and Soft Computing, 102, 370-377, (2016)

Piña, E., Cacería de Cargas. 1aㅡ edición, La ciencia Para Todos, Fondo de Cultura Económica. México D.F. (1995)

Piña, M., La Física en la Medicina. 1aㅡ edición, La ciencia Para Todos, Fondo de Cultura Económica. México D.F. (1998)

Polman, J. y Gebre, E., Towards critical appraisal if inforgraphics as a scientific inscription, doi: 10.1002/tea.21225, Journal of Research in Science Teaching, 52(6), 868-893. (2015)

Probosari, R.M., Widyastuti, F., y otros tres autores, Students' argument style through scientific reading-based inquiry: Improving argumentation skill in higher education, doi: 10.1063/1.5139820, AIP Conference Proceedings 2194, 020088 (2019) 
Reis, J.C., Guerra, A., Braga, M., y Freitas, J., History, Science and Culture: Curricular Experiences in Brazil, doi: 10.1023/A:1011289614116, Science \& Education, 10, 369-378 (2001)

Rivas, M. y Telleira, M. La literatura como estrategia de enseñanza de las ciencias naturales y matemática. Revista enseñanza de las ciencias, Número Extra VIII Congreso Internacional sobre Investigación en Didáctica de las Ciencias, Barcelona, 2760-2764 (2009)

Souza, J., Infographics: Ways of Seeing and Reading Science in Media, doi: 10.1590/2176-457323502, Bakhtiniana: Revista de Estudos do Discurso, 11(2), 190-206, (2016)

Valderrama, A.J. y Valderrama, O.J., Trabajo semestral guiado: un buen complemento al estudio y aprendizaje en cursos de Ciencia y de Ingeniería, doi: 10.4067/S0718-50062014000300002, Formación Universitaria, 7(3), 3-10 (2014) 